\title{
Transversus Abdominis Plane Block Appears to Be Effective and Safe as a Part of Multimodal Analgesia in Bariatric Surgery: a Meta-analysis and Systematic Review of Randomized Controlled Trials
}

\author{
Mária Földi $^{1,2,3}$. Alexandra Soós ${ }^{1,2,3} \cdot$ Péter Hegyi ${ }^{1,2,4} \cdot$ Szabolcs Kiss $^{1,2,3} \cdot$ Zsolt Szakács $^{1,2} \cdot$ Margit Solymár $^{1,2}$. \\ Erika Pétervári $^{1,2} \cdot$ Márta Balaskó ${ }^{1,2} \cdot$ Krzysztof Kusza $^{5} \cdot$ Zsolt Molnár $^{1,2,5}$ (D)
}

Received: 23 June 2020 / Revised: 7 September 2020 / Accepted: 10 September 2020 / Published online: 21 October 2020

(C) The Author(s) 2020

\begin{abstract}
Purpose Pain after bariatric surgery can prolong recovery. This patient group is highly susceptible to opioid-related side effects. Enhanced Recovery After Surgery guidelines strongly recommend the administration of multimodal medications to reduce narcotic consumption. However, the role of ultrasound-guided transversus abdominis plane (USG-TAP) block in multimodal analgesia of weight loss surgeries remains controversial.

Materials and Methods A systematic search was performed in four databases for studies published up to September 2019. We considered randomized controlled trials that assessed the efficacy of perioperative USG-TAP block as a part of multimodal analgesia in patients with laparoscopic bariatric surgery.

Results Eight studies (525 patients) were included in the meta-analysis. Pooled analysis showed lower pain scores with USGTAP block at every evaluated time point and lower opioid requirement in the USG-TAP block group (weighted mean difference $(\mathrm{WMD})=-7.59 \mathrm{mg} ; 95 \% \mathrm{CI}-9.86,-5.39 ; p<0.001)$. Time to ambulate was shorter with USG-TAP block $(\mathrm{WMD}=-2.22 \mathrm{~h}$; $95 \% \mathrm{CI}-3.89,-0.56 ; p=0.009)$. This intervention also seemed to be safe: only three non-severe complications with USG-TAP block were reported in the included studies.

Conclusion Our results may support the incorporation of USG-TAP block into multimodal analgesia regimens of ERAS protocols for bariatric surgery.
\end{abstract}

Keywords Pain $\cdot$ Bariatric surgery $\cdot$ TAP block $\cdot$ Meta-analysis

Electronic supplementary material The online version of this article (https://doi.org/10.1007/s11695-020-04973-8) contains supplementary material, which is available to authorized users.

\section{Zsolt Molnár}

zsoltmolna@gmail.com; zsolt.lajos.molnar@aok.pte.hu

1 Institute for Translational Medicine, Medical School, University of Pécs, 12 Ifjúság St., Pécs 7624, Hungary

2 Szentágothai Research Centre, University of Pécs, Pécs, Hungary

3 Doctoral School of Clinical Medicine, University of Szeged, Szeged, Hungary

4 Division of Translational Medicine, First Department of Medicine, Medical School, University of Pécs, Pécs, Hungary

5 Department of Anesthesiology and Intensive Therapy, Poznan University for Medical Sciences, Poznan, Poland

\section{Introduction}

Pain in the postoperative period can cause serious suffering to patients, prolong recovery, and increase healthcare costs [1]. However, postoperative pain management can be a major challenge as previous studies demonstrated that it is frequently suboptimal [2-4].

Laparoscopic bariatric surgeries are considered minimally invasive, but they can cause severe pain $[5,6]$. Opioids are excellent analgesics, but they have several side effects such as respiratory depression, which may further complicate pain management in weight loss surgeries, particularly in cases with obstructive sleep apnea [7]. Other comorbidities such as diabetes mellitus and cardiovascular diseases that are common in patients with obesity can also lead to difficulties with pain management [8]. This complexity highlights the 
importance and the challenges of the optimal choice of analgesia in bariatric surgery.

Enhanced Recovery After Surgery (ERAS) protocols are created to facilitate faster recovery after surgery multimodal analgesia [9]. Although growing evidence supports multimodal analgesic techniques in clinical practice, opioids still remain among the first choice of postoperative pain management [10].

Postoperative opioid overuse could be particularly worrisome. For example, in the USA, the opioid epidemic causes a serious health crisis. According to a recent study, persistent opioid use is a common problem after surgery [11]. In the opioid epidemic era, recognizing the issue of opioid overuse with its associated complications could be of particular importance [12]. Several alternative options can be used including other pain medications such as paracetamol, non-steroidal anti-inflammatory drugs (NSAIDs), ketamine, or gabapentin [13].

Besides pharmacological analgesia, locoregional analgesic techniques are also among the alternatives. After decades of being the "gold standard," large meta-analyses and trials reported controversial effects of epidural analgesia on mortality and morbidity associated with frequent technical failures [14, 15]. As an alternative to epidural analgesia, infiltrative techniques-including transversus abdominis plane block (TAP block) - has gained increasing attention in recent years as they can be safely and easily applied [16]. During TAP block, a local anesthetic solution is injected between planes of abdominal muscles to anesthetize the anterior abdominal wall [17]. As ultrasound guidance (USG) becomes more widely available, the popularity of TAP block has further increased. USG facilitates the performance of TAP block in cases where anatomic landmarks are poorly defined, e.g., in patients with obesity [18].

Recent meta-analyses showed that USG-TAP block is effective in reducing pain and opioid consumption in different abdominal surgeries [19], including open appendectomy [20], hysterectomy [21], or colorectal resection [15] to control pain and decrease opioid consumption. Randomized controlled studies (RCTs) investigating the use of TAP block in weight loss surgeries have also been published, but its impact on different outcomes remained controversial. To our knowledge, no meta-analysis has examined TAP block during laparoscopic bariatric surgery. Therefore, we aimed to assess the effects of USG-TAP block as a part of multimodal analgesia for postoperative pain management in patients undergoing laparoscopic bariatric surgery.

\section{Methods and Materials}

We report this systematic review and meta-analysis following the Preferred Reporting in Systematic Reviews and Meta- analyses (PRISMA) (Supplementary Material) [22]. We registered the protocol on PROSPERO under registration number CRD42020154384.

\section{Eligibility Criteria}

We included full-text RCTs that assessed the efficacy of perioperative USG-TAP block in postoperative analgesia compared with no treatment or sham intervention in patients who underwent laparoscopic bariatric surgery.

The following outcomes were analyzed: pain scores measured by the Visual Analog Scale (VAS) or the Numbering Rating Scale (NRS) on a scale from 0 to 10 within the first 24 postoperative hours, morphine requirement $(\mathrm{mg})$ within the first 24 postoperative hours, rate of nausea during phase I recovery, time to ambulate (hours), length of hospital stay (hours), operation time (hours).

\section{Search Strategy}

A systematic search was carried out in the following databases for studies published up to September 2019: CENTRAL, MEDLINE, Web of Science, and Embase. We designed a search key with synonyms to bariatric surgery (population) and TAP (intervention) linked with Boolean operators. We did not use any filters (e.g., language, full-text, human) (Supplementary Material). The reference lists of included studies and previous systematic reviews and meta-analyses have also been screened for additional articles. Gray literature was not included in our meta-analyses.

\section{Selection Strategy and Data Extraction}

Two authors independently (SK and MF) removed all duplicate records, then checked titles and abstracts to remove irrelevant articles, and evaluated full-text articles, whether they were eligible for inclusion. All disagreements were resolved by consensus.

Two authors independently (MF and SK) extracted data into a standardized data collection sheet. We resolved any disagreement by consensus. From the individual studies, we extracted the raw data (mean and standard deviation or standard error) in case of cumulative morphine dose, time to ambulate, length of hospital stay, operation time, and pain level in rest and at movement if it was given. In the case of nausea, the number of patients and event rates in the two groups were extracted from the individual studies.

\section{Risk of Bias Assessment}

Two independent authors (MF and SK) used the revised Cochrane risk-of-bias 2 (RoB 2) tool to assess the risk of bias 


\section{PRISMA 2009 Flow Diagram}

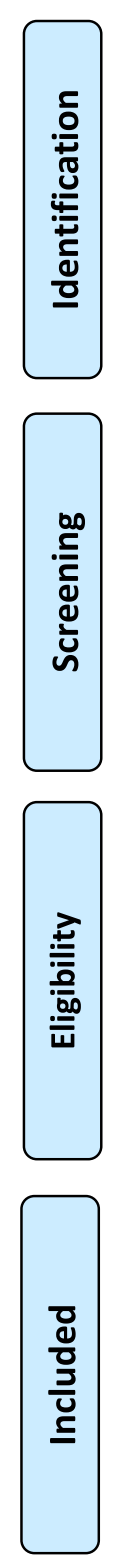

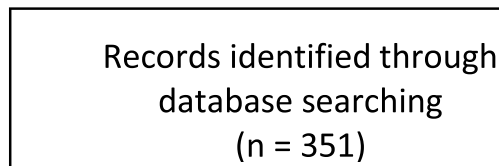

$(n=351)$

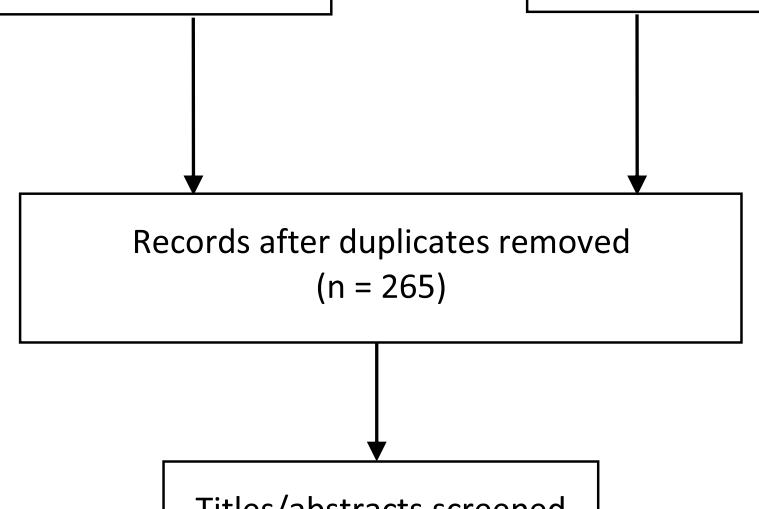

Full-text articles included from reference list screening $(n=1)$
Records excluded $(n=247)$

Full-text articles excluded, with reasons

$$
(n=10)
$$

other control group (5) other type of surgery or intervention (2) review or non-RCT (2) duplicate (1)

Fig. 1 Flow chart of study selection and inclusion process

of studies in the following categories [23]. Disagreements were resolved by consensus.

\section{Statistical Analysis}

We calculated mean differences with $95 \%$ CI between the control and USG-TAP groups. In the case of nausea, we calculated risk ratio with $95 \%$ CI. A $p$ value $<0.05$ was considered statistically significant. Pooled estimates were calculated with a random effects model by using the DerSimonianLaird method [24]. If mean with standard deviation was not reported, we estimated them from median, interquartile, and range [25]. Results of the meta-analysis were displayed graphically using forest plots. Due to methodological characteristics 


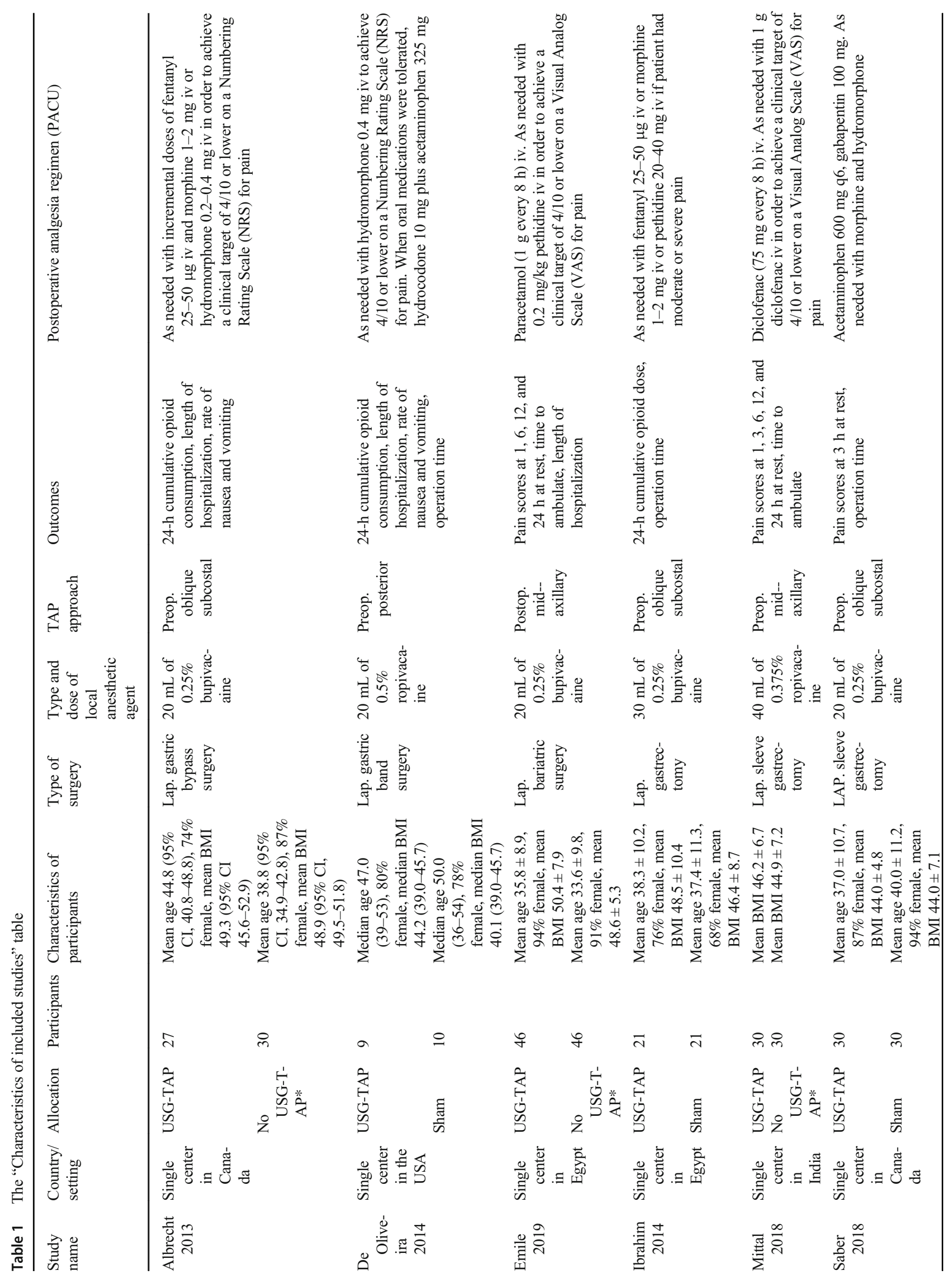




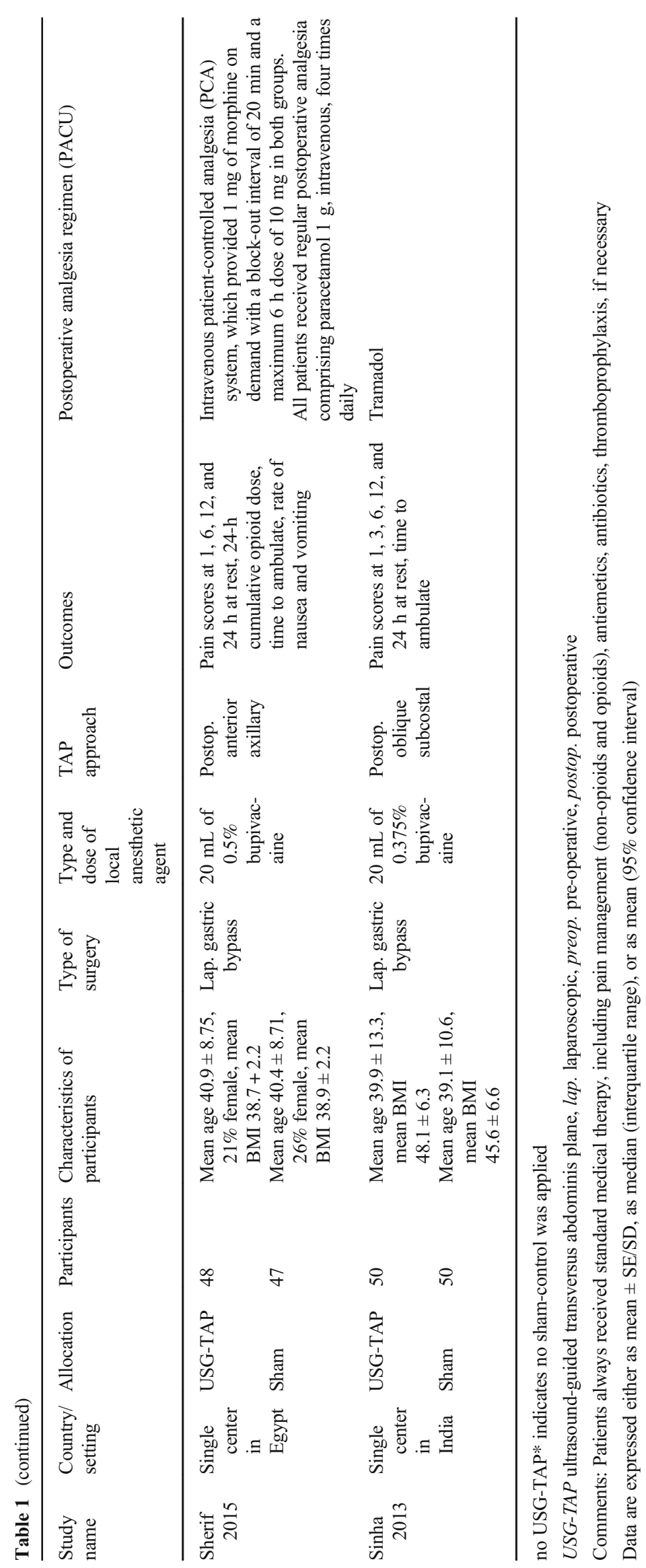




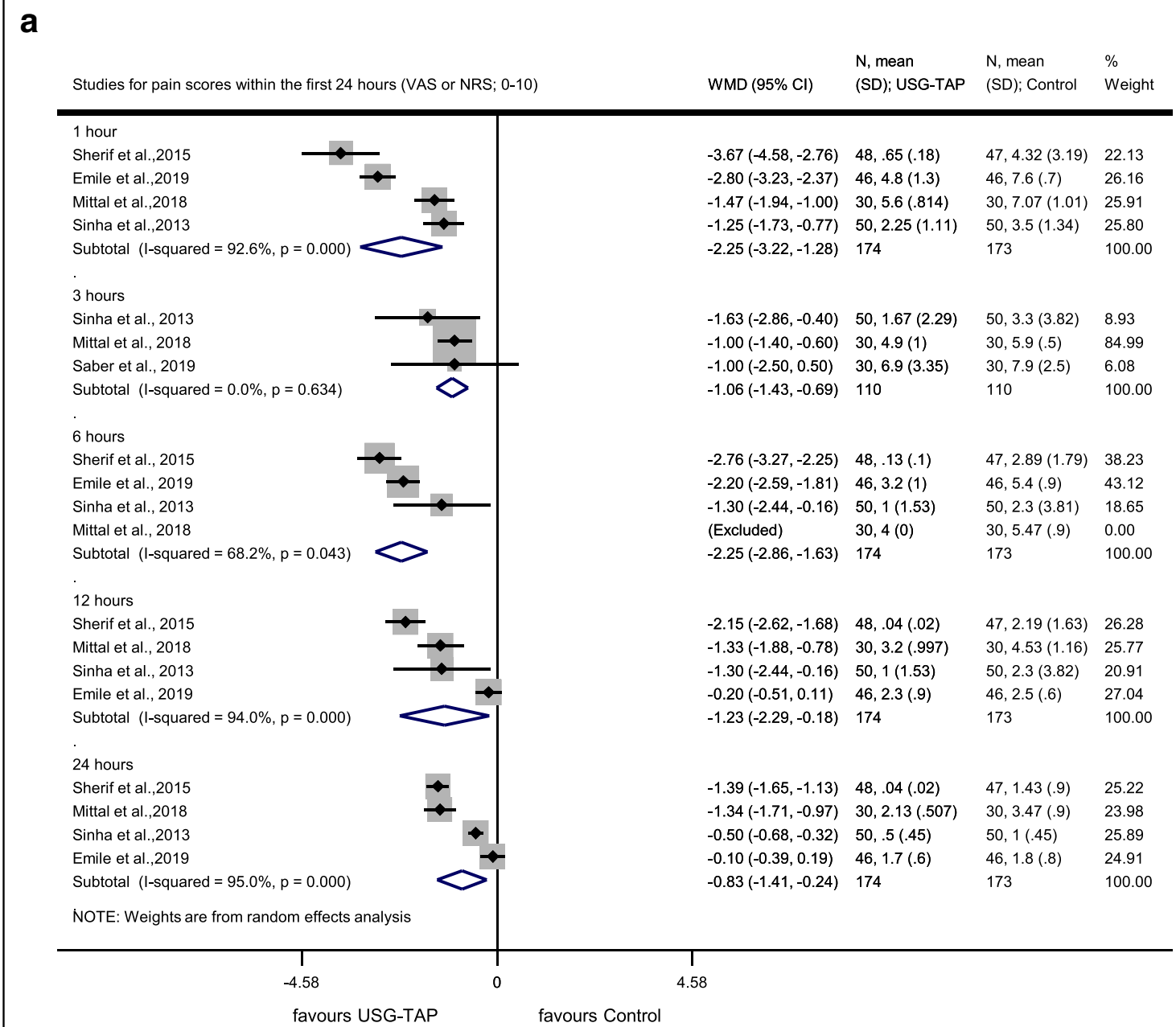

b

Studies for 24-hour morphine requirement (mg)

WMD $(95 \% \mathrm{Cl})$

$\mathrm{N}$, mean

$\mathrm{N}$, mean $\quad \%$

Studies for 24 -

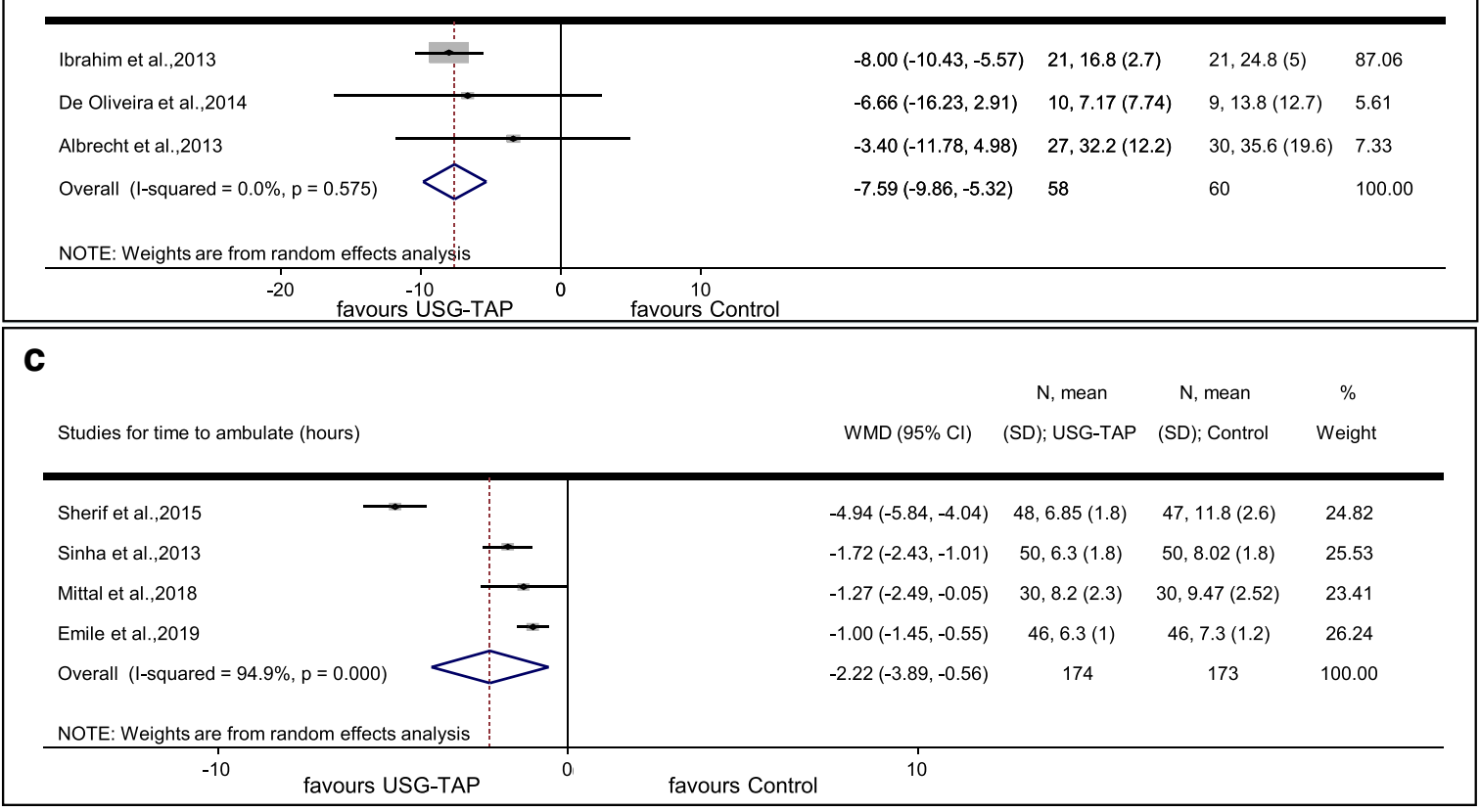

(SD); USG-TAP

(SD); Control Weight 
Fig. 2 Forest plots that show efficacy endpoints for the comparison of "USG-TAP" and "control". a Forest plot for pain score within the first 24 postoperative hours (VAS or NRS, 0-10). b Forest plot showing 24-h postoperative morphine requirement $(\mathrm{mg})$. $\mathbf{c}$ Forest plot showing time to ambulate (h). USG-TAP, ultrasound-guided transversus abdominis plane block; VAS, Visual Analog Scale; NRS, Numbering Rating Scale

of the analysis, we could not indicate pooled means for each group on the forest plots; however, study-level data in each study can be seen in Supplementary Material (for 24-h cumulative morphine requirement, time to ambulate, length of hospital stay, and operation time).

Heterogeneity was tested by using the Cochrane's $Q$ and the $I^{2}$ statistics, where $I^{2}=100 \% \times(Q-\mathrm{df}) / Q$, and represents the magnitude of the heterogeneity (moderate: $30-60 \%$, substantial: $50-90 \%$, considerable: $75-100 \%$ ) [16]. A $p$ value < 0.10 was considered statistically significant heterogeneity. All meta-analytical calculations were performed by Stata 11 data analysis and statistical software (Stata Corp LLC, College Station, TX, USA).

We performed trial sequential analysis (TSA) for each outcome if it was possible. We used the TSA tool to estimate the required number of patients in future studies and to quantify the statistical reliability of data if the condition of the tests were met. With this test, we assessed whether the intervention arm is effective applying adjusted significance tests and determined the necessity of conducting more studies in the topic to show significant differences [26].

We planned to conduct the following subgroup analyses: gender, age, type of bariatric surgery, type and dose of local anesthetics, TAP approach. Because of the limited number of studies, we were unable to conduct any of the planned subgroup analyses.

\section{Quality of Evidence}

We assessed the overall quality of evidence using the GRADE profiler (GRADEpro). Since data come from only RCTs, we downgraded the evidence from "high quality" by one level for serious (or by two levels for very serious) risk of bias, indirectness of evidence, serious inconsistency, imprecision of effect estimates, or potential publication bias.

We included the critical and important outcomes in the "Summary of findings table" (Table 2).

\section{Results}

\section{Results of Search and Selection}

The selection process is described in detail in the PRISMA flow diagram (Fig. 1). A total of 351 records were identified through electronic database search (CENTRAL: 89;
MEDLINE: 36; Web of Science: 99; Embase 127), eight of which were included in this meta-analysis $(n=525 ; 262$ in the "USG-TAP block" group and 263 in the "control" group). Beyond the eight analyzed articles, two studies with active control groups were excluded [27, 28], and in one excluded study, USG-TAP was not performed perioperatively [29].

\section{Characteristics of the Studies Included}

All included studies were single-center RCTs (Table 1). From the eight studies, five used sham-control (normal saline infiltration) [30-34]. In three studies, the control group did not receive sham-control [35-37]. One study used port-site infiltration in both intervention and control groups [37].

Studies reported data of patient group numbers ranging from 19 to 100 . Studies enrolled predominantly women with a mean BMI over 40 [33]. Four studies reported data of patients undergoing laparoscopic sleeve gastrectomy [31-33, 36]. Two studies recruited patients who underwent laparoscopic gastric bypass surgery [34, 37]. One trial studied patients with gastric band surgery [30] and one with several different types of laparoscopic bariatric surgery [35].

The type and dose of local anesthetic agents and those of USG-TAP approaches were different among studies. In four of the studies, USG-TAP block was performed immediately after completion of surgery [30-32, 37]; the remaining studies carried out surgeries with preoperative USG-TAP block after anesthesia induction [33-36].

Postoperative analgesia regimens were also quite diverse among studies (see in detail in Table 1); most of the studies used regular or as-needed non-opioids supplemented with narcotics on demand. However, some studies - carried out in the early 2010 - applied opioids exclusively [31, 34].

\section{Effects of Intervention}

\section{Primary Endpoints}

Pain Scores Within the First $48 \mathrm{~h}$ Pooled analysis showed that USG-TAP block lowered postoperative pain scores (rated on a scale between 0 and 10$)$ at rest by $2.25(p<0.001)$ at $1 \mathrm{~h}$, by $1.08(p<0.001)$ at $3 \mathrm{~h}$, by $2.25(p<0.001)$ at $6 \mathrm{~h}$, by 1.23 $(p<0.022)$ at $12 \mathrm{~h}$, and by $0.83(p=0.006)$ at $24 \mathrm{~h}$ (Fig. $2 \mathrm{a})$. Heterogeneity was considerable in these analyses (Fig. 2a).

Two studies also examined pain scores at rest $48 \mathrm{~h}$ after surgery: they found significantly lower pain scores in the USG-TAP block group [33, 36].

In two included studies $[33,36]$, pain scores at movement were also significantly lower at each evaluated time point $(0.5$, $3,6,12,24$, and $48 \mathrm{~h}$ postoperatively; $p<0.001$ for all comparisons). 
Postoperative Cumulative Morphine Dose Four studies with 213 patients (106 in the intervention group and 107 in the control group) examined the postoperative cumulative morphine dose within the first $24 \mathrm{~h}$ [30, 31, 33, 37]. Morphine requirement did not differ significantly between the intervention and control groups ( $-12 \mathrm{mg} ; 95 \% \mathrm{CI}-26.88,2.89 ; p=$ $0.114)$. However, we observed high heterogeneity in this analysis $\left(p_{\text {heterogeneity }}<0.001\right.$ and $\left.I^{2}=99.0 \%\right)$. We identified and removed the influential study with sensitivity analysis, which reduced heterogeneity to $0 \%$ and changed a direction of the main association to favoring TAP (Fig. 2b) [33]. Results of each study can be seen in Supplementary Material.

\section{Secondary Endpoints}

Time to Postoperative Bowel Recovery One trial with 46 patients in each arm reported recovery of bowel functions assessed by time to first flatus, and they found a statistically significant difference favoring the USG-TAP block group $(9.5 \pm 1.9$ vs $10.5 \pm 2.2 \mathrm{~h} ; p<0.001)$ [35]. Mittal and coworkers also found earlier resumption of bowel activity in the intervention group [36].

Nausea and Vomiting Pooled analyses of three studies with 171 patients ( 85 in the intervention and 86 in the control groups) indicated a lower risk of nausea in the USG-TAP block groups compared with control patients $(95 \% \mathrm{CI}, \mathrm{RR}=$ $0.24, p<0.001$ ) (Supplementary Material) [30, 31, 33, 37].

Emile and coworkers applied the Apfel score for postoperative nausea and vomiting: they also found a significant improvement with USG-TAP block for this outcome $(2.1 \pm 0.9$ points in the USG-TAP group vs $3.0 \pm 0.9$ points, $p<0.001$ in the control group) [35]. Mittal and coworkers reported a pooled number of events of nausea and/or vomiting and found $8 / 30$ and 24/30 cases in the USG-TAP and control groups, respectively [36].

However, both Emile et al. and Saber et al. found that the need for antiemetic use was similar between intervention and control groups [32, 35].

Sedation In the study of Sherif et al., four patients of 47 in the control group required postoperative biphasic intermittent positive airway pressure (BIPAP) ventilation support [33]. According to the study of Sinha et al., four of 50 patients needed BIPAP in the control group [34]. None of these studies detected any need for BIPAP in the USG-TAP group.

Sinha and coworkers also reported significantly lower Richmond Agitation and Sedation Score in the first 6 hours in the USG-TAP block group [34].

Time to Ambulate Pooled analysis of four trials with 347 patients (174 in the intervention group and 173 in the control group) demonstrated that the time to ambulate was shorter by
$2.2 \mathrm{~h}$ in patients who underwent USG-TAP block $(p=0.009)$ (Fig. 2c) [33-36]. We observed high heterogeneity for this meta-analysis (Fig. 2c). After sensitivity analysis, we identified an influential study [34]. Removal of this study changed the result to non-significant; however, heterogeneity remained high (weighted mean difference $(\mathrm{WMD})=-2.40 ; 95 \% \mathrm{CI}-$ 4.98, 0.18; $p<0.001$ ( $p_{\text {heterogeneity }}<0.001$ and $\left.I^{2}=96.6 \%\right)$ ). (Results of each study are shown in Supplementary Material.)

Length of Hospital Stay A meta-analysis of three studies with 168 patients ( 83 in the intervention group and 85 in the control group) failed to identify a shorter length of hospital stay following USG-TAP block performance compared with that of controls $(p=0.102)$ (Supplementary Material) [30, 35, 37]. (Results of each study are shown in Supplementary Material.)

Length of Operation Three studies with 121 patients (61 in the intervention group and 60 in the control group) using preoperative USG-TAP block evaluated the length of operation. We found similar operative times in the intervention and control groups ( $p=0.951)$ (Supplementary Material) [30-32]. (Results of each study are shown in Supplementary Material.)

Satisfaction Rate Two studies investigated the patient satisfaction rate with different methods. In the study of Mittal and coworkers, it was assessed by the Capuzzo composite score (score range 0-10) in 60 patients: the authors reported significantly higher scores in the USG-TAP block group compared with the control group $(8.2 \pm 0.7$ vs $7.1 \pm 0.7 ; p<0.001)$ [36]. Sinha and coworkers also observed significantly higher satisfaction scores in the USG-TAP block group at the end of the first postoperative day [34].

USG-TAP Block-Related Complications Only three occurrences of local complications (two cases with hematoma formation, one case with severe pain at the site of injection) due to USG-TAP block were reported in only one study [35].

\section{Trial Sequential Analysis}

The cumulative $Z$ curve crossed trial sequential significance boundary with regard to the outcomes: time to ambulate, nausea and vomiting, pain at 1 and $24 \mathrm{~h}$. In addition, nausea and vomiting and pain at $1 \mathrm{~h}$ exceeded the required meta-analysis sample size, from which it can be inferred that inclusion of further clinical trials would not change these results (Fig. S5). TSA for morphine requirement and operation time could not be performed due to insufficient availability of data.

\section{Risk of Bias in the Studies Included}

We summarized the results of the risk of bias assessment for each included study in Fig. 3 and Fig. S6. 
Fig. 3 Risk of bias summary: review authors' judgments about each risk of bias item for each included study

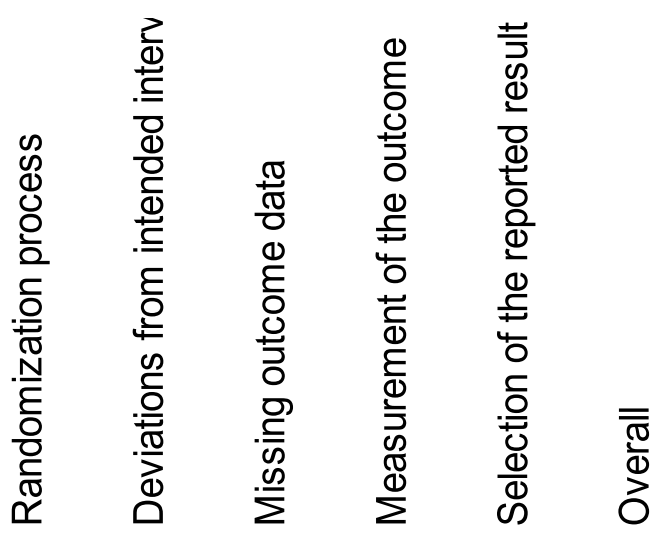

Albrecht, 2013
De Oliveira, 2014
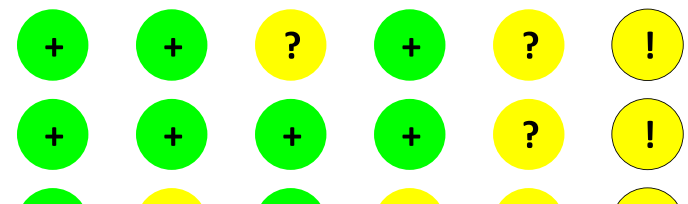

Emile, 2019

Ibrahim, 2014

Mittal, 2018

Saber, 2019

Sherif, 2015

Sinha, 2013
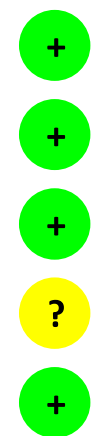

(-)

$+$
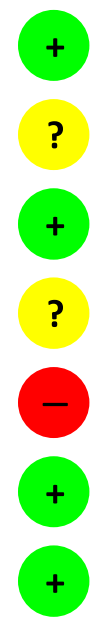

$+$

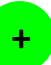

?
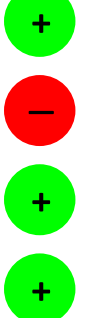
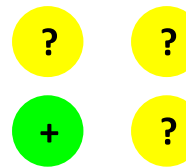

? ?
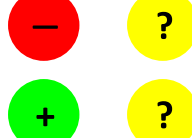

?

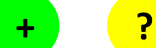

$?$

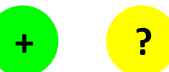

\section{Discussion}

This meta-analysis and systematic review investigates the efficacy and safety of USG-TAP block compared with systemic analgesia alone in patients undergoing laparoscopic bariatric surgery. Our analyses suggest various beneficial effects, including a reduction in pain scores, in opioid requirement, and in risk for adverse events associated with opioids in the first 24 postoperative hours, without any reported major adverse events.

We detected a statistically significant decrease in resting pain scores at each evaluated time point during the first 24 postoperative hours. Included studies assessed pain intensity by Visual Analog Scale (VAS) or Numbering Rating Scale (NRS) on a scale from 0 to 10. Previously, Kelly and coworkers reported that the minimum clinically significant difference in VAS score is 0.9 [38]. Accordingly, our results may also demonstrate clinically significant improvement, except for the 24th-hour postoperative pain scores, where we found only 0.83 lower WMD in the USG-TAP block group. Two studies also reported a beneficial effect of USG-TAP block on pain $48 \mathrm{~h}$ after surgery: the difference between groups was still statistically significant, but it gradually decreased with time $[33,36]$.

Interestingly, although the mean elimination half-life of bupivacaine is around 8-10 h after USG-TAP block [39], our results hint at a somewhat longer analgesic effectiveness in agreement with previous studies [21, 40], USG-TAP block appears to be effective in late pain as well but to a lesser extent. We evaluated our findings with some reservations because of the low quality of evidence due to inconsistency and the moderate/high risk of bias in individual studies (Table 2).

Meta-analysis of four RCTs on cumulative morphine requirement in the first $24 \mathrm{~h}$ showed a tendency favoring USG- 
Table 2 Summary of findings table. Patient or population: postoperative pain management in obese patients undergoing laparoscopic bariatric surgery; Setting: inpatient; Intervention: transversus abdominis plane block (TAP block) as a part of multimodal analgesia; Comparison: systemic analgesia alone (no intervention or sham-control)

\begin{tabular}{|c|c|c|c|}
\hline Outcomes & $\begin{array}{l}\text { № of participants } \\
\text { (studies) follow-up }\end{array}$ & $\begin{array}{l}\text { Certainty of the } \\
\text { evidence (GRADE) }\end{array}$ & $\begin{array}{l}\text { Risk difference with transversus abdominis plane block (TAP } \\
\text { block) as a part of multimodal analgesia }\end{array}$ \\
\hline $\begin{array}{l}\text { Pain score } 1 \mathrm{~h} \text { after surgery } \\
\text { assessed with VAS or NRS }\end{array}$ & 347 (4 RCTs) & $\oplus \oplus \circ \circ$ Low $^{\mathrm{a}, \mathrm{b}}$ & MD 2.25 lower (3.22 lower to 1.28 lower) \\
\hline $\begin{array}{l}\text { Pain score } 24 \mathrm{~h} \text { after surgery } \\
\text { assessed with VAS or NRS }\end{array}$ & 347 (4 RCTs) & $\oplus \oplus \circ \bigcirc$ Low $^{\mathrm{a}, \mathrm{b}}$ & MD 0.83 lower ( 1.41 lower to 0.24 lower) \\
\hline $\begin{array}{l}\text { 24-h postoperative cumulative } \\
\text { morphine dose (mg) }\end{array}$ & 118 (3 RCTs) & $\oplus \oplus \oplus \circ$ Moderate $^{c}$ & MD $7.59 \mathrm{mg}$ lower (9.86 lower to 5.32 lower) \\
\hline $\begin{array}{l}\text { Local and systemic complication } \\
\text { due to TAP block }\end{array}$ & 525 (8 RCTs) & - & Not pooled \\
\hline Time to ambulate $(\mathrm{h})$ & 347 (4 RCTs) & $\oplus \oplus \circ \circ$ Low $^{\mathrm{a}, \mathrm{b}}$ & MD $2.2 h$ fewer ( 3.89 fewer to 0.56 fewer) \\
\hline
\end{tabular}

*The risk in the intervention group (and its $95 \%$ confidence interval) is based on the assumed risk in the comparison group and the relative effect of the intervention (and its $95 \% \mathrm{CI}$ )

$C I$ confidence interval, $M D$ mean difference

GRADE Working Group grades of evidence:

High certainty: We are very confident that the true effect lies close to that of the estimate of the effect.

Moderate certainty: We are moderately confident in the effect estimate: The true effect is likely to be close to the estimate of the effect, but there is a possibility that it is substantially different.

Low certainty: Our confidence in the effect estimate is limited: The true effect may be substantially different from the estimate of the effect.

Very low certainty: We have very little confidence in the effect estimate: The true effect is likely to be substantially different from the estimate of effect.

${ }^{a}$ In a single study, there was no information of allocation concealment. In two studies, lack of blinding could lead to bias

${ }^{\mathrm{b}}$ Heterogeneity was high for this analysis

${ }^{\mathrm{c}}$ Optimal information size is not met calculated by trial sequential analysis

TAP block, albeit with a high heterogeneity. After removing the influential study verified by sensitivity analysis, heterogeneity disappeared, and the difference became significant.

We speculate that this phenomenon can be due to the much larger intergroup difference in morphine consumption in the influential study compared with the other studies [33]. This might result from the dissimilar study population (predominantly male and leaner patients) and the use of patientcontrolled analgesia, unlike in the other trials. It is important to note that although ERAS guidelines recommend patientcontrolled administration of opioids, only one study used patient-controlled analgesia (PCA) [9, 33]. We downgraded this outcome to moderate quality of evidence because it was not supported by a large enough data pool (Table 2).

Previous findings in the literature are controversial with regard to the effect of USG-TAP block on morphine requirement. Most studies agree that TAP block reduces opioid requirement in lower [24] and upper abdominal surgeries (as compared with placebo or no intervention) [40]. However, when TAP block was compared with or added to epidural analgesia [41], intrathecal analgesia [42], or wound infiltration in abdominal surgeries [43], there was usually no difference between groups. These findings may suggest that TAP block has no superior or added effect to these techniques in different types of abdominal surgeries. However, some studies have demonstrated the benefits of adding TAP block to infiltration of port sites [44], or even the superiority of TAP block over wound infiltration in general surgery [45].

One of the analyzed studies performed port-site infiltration in both USG-TAP and control groups; this is the only study which did not find significantly reduced morphine consumption in the USG-TAP block group [37]. In contrast, when Ruiz-Tovar and coworkers compared laparoscopic-guided TAP block directly with port-site infiltration in Roux-en-Y gastric bypass surgery, they could demonstrate the superiority of USG-TAP block over port-site infiltration [27]. Based on these findings, it appears that TAP block may lack an added effect to local infiltration anesthesia in bariatric surgery, but it appears to be preferable over local infiltration techniques. Since a definitive conclusion on the comparison of these two methods has not been reached, this topic in both bariatric and other abdominal surgeries would warrant further studies [46].

Enhanced Recovery After Surgery (ERAS) guidelines strongly recommend the administration of multimodal intravenous medication accompanied by local anesthetic infiltration in order to spare or avoid narcotic consumption in a patient group which is highly susceptible to the adverse events of opioids [9]. Nausea, vomiting, constipation, excessive sedation, and respiratory depression may prolong recovery, cause additional complications, and impair satisfaction rate of 
patients. Previous studies showed that multimodal analgesia reduces the rate of side effects and the time to recovery [47].

Our review discusses thoroughly the effects of USG-TAP block on opioid-related harms; USG-TAP block seems to be beneficial in each evaluated aspect (time to postoperative bowel recovery, nausea and vomiting, sedation). However, we could not reach a strong conclusion based on these results, because the pooled analysis was only possible in the case of nausea and vomiting indicating $76 \%$ relative risk reduction, and the 1-h reduction in time to first flatus was on the one hand reported by only one study, and on the other, its clinical relevance is questionable despite the statistically significant result [35].

Our meta-analysis indicates shorter time required to ambulate with USG-TAP block. This may support faster recovery and a reduced number of complications of immobilization. Since both obesity and postoperative conditions are risk factors of thromboembolism, patients with bariatric surgery are at a particularly high risk for these complications [48]. Besides thromboprophylaxis, decreasing length of bed rest can be an important factor in thrombosis prevention. We downgraded this outcome to low quality of evidence because of inconsistency and risk of bias (Table 2).

The presence of USG-TAP block did not affect the total length of hospital stay, even if we would expect that early ambulation would be associated with faster discharge [49]. Nevertheless, since the length of hospital stay depends on several factors, and patients spent only about 2 days in hospital, minor differences might have remained undetected. Further studies assessing the length of hospital stay as the primary outcome could resolve this issue.

TAP block is usually considered safe, but rare complications such as puncture of the liver may occur [50]. Among studies included in this review, only Emile and coworkers reported two cases of abdominal wall hematoma and one case of severe pain at the site of injection [35]. Of course, there are more appropriate study designs to detect rare side effects than RCTs, which could not be included in the current metaanalysis as they did not fit in the inclusion criteria. In the future, it would be important to record complications more thoroughly in RCTs.

Despite the previous concerns regarding challenges to TAP block administration in patients with obesity [51], only two studies mentioned minor difficulties that were successfully eliminated [31, 34]. In addition, we incorporated only those trials that operated under ultrasound guidance, which facilitates better visualization. However, most of the included studies failed to report success rates.

Heterogeneity was high between studies. Since the low number of analyzed studies did not allow subgroup analyses, we were not able to explore the cause of heterogeneity - with one exception mentioned above. Theoretically, we can explain heterogeneity by the different types of surgery, anesthetic management, dose and type of anesthetics, USGTAP approach, or postoperative analgesia regimen.

It is well known that USG-TAP block relieves somatic but not visceral pain. The ratio of pain types can differ depending on the types of bariatric surgery, affecting the extent of USGTAP block efficacy, as well. A cadaver study has suggested that the subcostal approach is superior to the mid-axillary approach as indicated by the size of dye spread [52]. In addition, Khan et al. and coworkers achieved better postoperative analgesia with the subcostal approach in patients with cholecystectomy compared with the posterior approach [53]. Thus, the subcostal approach may be better when compared with other techniques in upper abdominal surgeries. It has been also suggested that the pre-incisional application of TAP block may be more potent than post-incisional application, because of the preemptive analgesia that spares patients from the development of altered processing of afferent input [54]. Since we could not perform subgroup analyses to address these questions, further well-designed clinical trials would be required.

In addition to high heterogeneity across studies, the poor reporting of important outcomes by relatively few, small, and single-center studies is another important limitation of our meta-analysis as well as the risk of bias of the included studies. The definition of some outcomes (e.g., operation time) was not precise enough. Conversion of medians to mean could distort our result. Some of the included studies may raise ethical concerns since they worked with invasive placebo (so-called shamcontrol). The SHAM (serious harm and morbidity) scale classifies the risk of saline injection as placebo control of TAP block as highest (grade 4) [55].

Further limitation can be that some studies were conducted before the "paradigm shift" in opioid use, which means that these studies might apply non-opioids inadequately. The combination of TAP block with non-opioid pain medication within the framework of opioid-restrictive protocols would worth further studying. The analgesic regimens were not only outdated in some studies but also very diverse across studies. For instance, pethidine was used as an opioid in one of the studies, which has become obsolete in several countries for more than two decades [35]. It is, therefore, challenging to compare "old fashioned" single-agent techniques to up-to-date multimodal approaches.

Further studies are also necessary to elucidate the optimal use of USG-TAP block in bariatric surgery, including the ideal timing, technique, dose, or type of local anesthetic injection. We also need to know more about its efficacy when it is added to or compared with other analgesic agents in order to find its place in multimodal analgesia. There are further promising fields in TAP block research as the use of continuous infusion of local anesthetics or liposomal bupivacaine. 


\section{Conclusion}

In summary, USG-TAP block reduces pain intensity, morphine requirement, rate of opioid-related side effects, and the time to ambulate. It is likely to help the faster recovery of patients, even if this meta-analysis could not detect significantly shorter length of hospital stay with USG-TAP block. Our results may support its incorporation into multimodal analgesia regimens of ERAS protocols for patients undergoing laparoscopic bariatric surgery, but further studies are needed to evaluate its co-administration with non-opioid medication in opioid-restrictive protocols.

Acknowledgments We would like to thank Professor Olle Ljungqvist (Professor of Surgery, Faculty of Medicine and Health, School of Health and Medical Sciences Department of Surgery Örebro University, Örebro, Sweden) for his useful comments and acting as an advisor in preparing the manuscript. This study was supported by the ÚNKP-203-New National Excellence Program of the Ministry for Innovation and Technology from the source of the National Research, Development and Innovation Fund.

Funding Open access funding provided by University of Pécs. This study was funded by "GINOP-2.3.2-15-2016-00048 - STAY ALIVE" cofinanced by the European Union (European Regional Development Fund) within the framework of Programme Széchenyi 2020 and by the Human Resources Development Operational Programme Grant, Grant Number: EFOP 3.6.2-16-2017-00006 - LIVE LONGER which is cofinanced by the European Union (European Regional Development Fund) within the framework of Programme Széchenyi 2020.

\section{Compliance with Ethical Standards}

Conflict of Interest The authors declare that they have no conflict of interest.

Ethical Approval Statement This article does not contain any studies with human participants or animals performed by any of the authors.

Informed Consent Statement Informed consent does not apply.

Open Access This article is licensed under a Creative Commons Attribution 4.0 International License, which permits use, sharing, adaptation, distribution and reproduction in any medium or format, as long as you give appropriate credit to the original author(s) and the source, provide a link to the Creative Commons licence, and indicate if changes were made. The images or other third party material in this article are included in the article's Creative Commons licence, unless indicated otherwise in a credit line to the material. If material is not included in the article's Creative Commons licence and your intended use is not permitted by statutory regulation or exceeds the permitted use, you will need to obtain permission directly from the copyright holder. To view a copy of this licence, visit http://creativecommons.org/licenses/by/4.0/.

\section{References}

1. Gamboa JSB. Pain management in weight loss surgery: aiming for multimodal approach. Adv Obes Weight Manag Control. 2016;5(2):00125.
2. Fletcher D, Fermanian C, Mardaye A, et al. A patient-based national survey on postoperative pain management in France reveals significant achievements and persistent challenges. Pain. 2008;137(2): 441-51.

3. Meissner W, Huygen F, Neugebauer EAM, et al. Management of acute pain in the postoperative setting: the importance of quality indicators. Curr Med Res Opin. 2018;34(1):187-96.

4. Sommer M, de Rijke JM, van Kleef M, et al. The prevalence of postoperative pain in a sample of 1490 surgical inpatients. Eur J Anaesthesiol. 2008;25(4):267-74.

5. Hartwig M, Allvin R, Backstrom R, et al. Factors associated with increased experience of postoperative pain after laparoscopic gastric bypass surgery. Obes Surg. 2017;27(7):1854-8.

6. Weingarten TN, Sprung J, Flores A, et al. Opioid requirements after laparoscopic bariatric surgery. Obes Surg. 2011;21(9):1407-12.

7. Mason M, Cates CJ, Smith I. Effects of opioid, hypnotic and sedating medications on sleep-disordered breathing in adults with obstructive sleep apnoea. Cochrane Database Syst Rev. 2015;(7): CD011090.

8. Gupta K, Prasad A, Nagappa M, et al. Risk factors for opioidinduced respiratory depression and failure to rescue: a review. Curr Opin Anaesthesiol. 2018;31(1):110-9.

9. Thorell A, MacCormick AD, Awad S, et al. Guidelines for perioperative care in bariatric surgery: Enhanced Recovery After Surgery (ERAS) Society recommendations. World J Surg. 2016;40(9): 2065-83.

10. Hill MV, McMahon ML, Stucke RS, et al. Wide variation and excessive dosage of opioid prescriptions for common general surgical procedures. Ann Surg. 2017;265(4):709-14.

11. Brummett CM, Waljee JF, Goesling J, et al. New persistent opioid use after minor and major surgical procedures in US adults. JAMA Surg. 2017;152(6):e170504.

12. $\mathrm{Wu} \mathrm{CL}$, King $\mathrm{AB}$, Geiger TM, et al. American Society for Enhanced Recovery and Perioperative Quality Initiative Joint Consensus statement on perioperative opioid minimization in opioid-naive patients. Anesth Analg. 2019;129(2):567-77.

13. Baboli KM, Liu H, Poggio JL. Opioid-free postoperative analgesia: is it feasible? Curr Probl Surg. 2020;57(7):100795.

14. Leslie K, Myles P, Devereaux P, et al. Neuraxial block, death and serious cardiovascular morbidity in the POISE trial. Br J Anaesth. 2013;111(3):382-90.

15. Liu L, Xie YH, Zhang W, et al. Effect of transversus abdominis plane block on postoperative pain after colorectal surgery: a metaanalysis of randomized controlled trials. Med Princ Pract. 2018;27(2):158-65.

16. Ma N, Duncan JK, Scarfe AJ, et al. Clinical safety and effectiveness of transversus abdominis plane (TAP) block in post-operative analgesia: a systematic review and meta-analysis. J Anesth. 2017;31(3):432-52.

17. McDonnell JG, O'Donnell BD, Tuite $\mathrm{D}$, et al. The regional abdominal field infiltration (R.A.F.I.) technique: computerised tomographic and anatomical identification of a novel approach to the transversus abdominis neuro-vascular fascial plane. Anesthesiology. 2004;101(A899)

18. Narouze SN, Guirguis M. Ultrasound-guided transversus abdominis plane (TAP) block. Atlas of ultrasound-guided procedures in interventional pain management. 2018. p. 157-60.

19. Johns N, O'Neill S, Ventham NT, et al. Clinical effectiveness of transversus abdominis plane (TAP) block in abdominal surgery: a systematic review and meta-analysis. Color Dis. 2012;14(10): e635-42.

20. Niraj G, Searle A, Mathews M, et al. Analgesic efficacy of ultrasound-guided transversus abdominis plane block in patients undergoing open appendicectomy. Br J Anaesth. 2009;103(4): $601-5$. 
21. Champaneria R, Shah L, Geoghegan J, et al. Analgesic effectiveness of transversus abdominis plane blocks after hysterectomy: a meta-analysis. Eur J Obstet Gynecol Reprod Biol. 2013;166(1):19.

22. Moher D, Liberati A, Tetzlaff J, et al. Preferred reporting items for systematic reviews and meta-analyses: the PRISMA statement. PLoS Med. 2009;6(7):e1000097.

23. Sterne JAC, Savovic J, Page MJ, et al. RoB 2: a revised tool for assessing risk of bias in randomised trials. BMJ. 2019;366:14898.

24. DerSimonian RLN. Meta-analysis in clinical trials. Control Clin Trials. 1986;7(3):177-88.

25. Wan XLJ, Tong T. Estimating the sample mean and standard deviation from the sample size median, range and/or interquartile range. BMC Med Res Methodol. 2014;14(1):135.

26. Thorlund K EJ, Wetterslev J, Brok J, Imberger G, Gluud C. User manual for trial sequantial analysis (TSA). Copenhagen Trial Unit, Centre for Clinical Intervention Research, Copenhaged, Denmark. 2011:1-115.

27. Ruiz-Tovar J, Garcia A, Ferrigni C, et al. Laparoscopic-guided transversus abdominis plane (TAP) block as part of multimodal analgesia in laparoscopic Roux-en-Y gastric bypass within an Enhanced Recovery After Surgery (ERAS) program: a prospective randomized clinical trial. Obes Surg. 2018;28(11):3374-9.

28. Said AM, Balamoun HA. Continuous transversus abdominis plane blocks via laparoscopically placed catheters for bariatric surgery. Obes Surg. 2017;27(10):2575-82.

29. Wassef M, Lee DY, Levine JL, et al. Feasibility and analgesic efficacy of the transversus abdominis plane block after single-port laparoscopy in patients having bariatric surgery. J Pain Res. 2013;6: 837-41.

30. De Oliveira Jr GS, Fitzgerald P, Ahmad S, et al. Transversus abdominis plane infiltration for laparoscopic gastric banding: a pilot study. World J Gastrointest Surg. 2014;6(2):27-32.

31. Ibrahim M, Shamaa HE. Efficacy of ultrasound-guided oblique subcostal transversus abdominis plane block after laparoscopic sleeve gastrectomy: a double blind, randomized, placebo controlled study. Egypt J Anaesth. 2014;30(3):285-92.

32. Saber AA, Lee YC, Chandrasekaran A, et al. Efficacy of transversus abdominis plane (TAP) block in pain management after laparoscopic sleeve gastrectomy (LSG): a double-blind randomized controlled trial. Am J Surg. 2019;217(1):126-32.

33. Sherif A, Koptan H, Soliman S. Feasibility and perioperative painrelieving efficacy of ultrasound-guided transversus abdominis plane block in morbidly obese patients undergoing laparoscopic bariatric surgery. Research and Opinion in Anesthesia and Intensive Care. 2015;1(1):50

34. Sinha A, Jayaraman L, Punhani D. Efficacy of ultrasound-guided transversus abdominis plane block after laparoscopic bariatric surgery: a double blind, randomized, controlled study. Obes Surg. 2013;23(4):548-53.

35. Emile SH, Abdel-Razik MA, Elbahrawy K, et al. Impact of ultrasound-guided transversus abdominis plane block on postoperative pain and early outcome after laparoscopic bariatric surgery: a randomized double-blinded controlled trial. Obes Surg. 2019;29(5):1534-41.

36. Mittal T, Dey A, Siddhartha R, et al. Efficacy of ultrasound-guided transversus abdominis plane (TAP) block for postoperative analgesia in laparoscopic gastric sleeve resection: a randomized single blinded case control study. Surg Endosc. 2018;32(12):4985-9.

37. Albrecht E, Kirkham KR, Endersby RV, et al. Ultrasound-guided transversus abdominis plane (TAP) block for laparoscopic gastricbypass surgery: a prospective randomized controlled doubleblinded trial. Obes Surg. 2013;23(8):1309-14.

38. Kelly. Does the clinically significant difference in visual analog scale pain scores vary with gender, age, or cause of pain? Acad Emerg Med. 1998;5(11):1086-90.
39. Trabelsi B, Charfi R, Bennasr L, et al. Pharmacokinetics of bupivacaine after bilateral ultrasound-guided transversus abdominis plane block following cesarean delivery under spinal anesthesia. Int J Obstet Anesth. 2017;32:17-20.

40. Peng K, Ji FH, Liu HY, et al. Ultrasound-guided transversus abdominis plane block for analgesia in laparoscopic cholecystectomy: a systematic review and meta-analysis. Med Princ Pract. 2016;25(3):237-46.

41. Baeriswyl M, Zeiter F, Piubellini D, et al. The analgesic efficacy of transverse abdominis plane block versus epidural analgesia: a systematic review with meta-analysis. Medicine (Baltimore). 2018;97(26):e11261.

42. Abdallah FW, Halpern SH, Margarido CB. Transversus abdominis plane block for postoperative analgesia after Caesarean delivery performed under spinal anaesthesia? A systematic review and meta-analysis. Br J Anaesth. 2012;109(5):679-87.

43. Nanze Yu XL, Jorge R, Lujan-Hernandez, et al. Transversus abdominis-plane block versus local anesthetic wound infiltration in lower abdominal surgery: a systematic review and metaanalysis of randomized controlled trials. Int J Obstet Anesth. 2017;32:17-20.

44. Pedrazzani C, Menestrina N, Moro M, et al. Local wound infiltration plus transversus abdominis plane (TAP) block versus local wound infiltration in laparoscopic colorectal surgery and ERAS program. Surg Endosc. 2016;30(11):5117-25.

45. Park JS, Choi GS, Kwak KH, et al. Effect of local wound infiltration and transversus abdominis plane block on morphine use after laparoscopic colectomy: a nonrandomized, single-blind prospective study. J Surg Res. 2015;195(1):61-6.

46. Yu NLX, Lujan-Hernandez JR, Succar J, et al. Transversus abdominis-plane block versus local anesthetic wound infiltration in lower abdominal surgery: a systematic review and metaanalysis of randomized controlled trials. BMC Anesthesiol. 2014;14(1):121

47. Omar SHRK, Youssif MA. A non opioid fast track anesthetic regimen for colonic resection. J Egypt Soc Parasitol. 2009;39(3):84964.

48. Stein PD, Matta F. Pulmonary embolism and deep venous thrombosis following bariatric surgery. Obes Surg. 2013;23(5):663-8.

49. Adogwa O, Elsamadicy AA, Fialkoff J, et al. Early ambulation decreases length of hospital stay, perioperative complications and improves functional outcomes in elderly patients undergoing surgery for correction of adult degenerative scoliosis. Spine (Phila Pa 1976). 2017;42(18):1420-5.

50. Lancaster PCM. Liver trauma secondary to ultrasound-guided transversus abdominis plane block. Br J Anaesth. 2010;104(4): $509-10$.

51. Karen C, Nielsen UG, Greengrass SM, et al. Influence of obesity on surgical regional anesthesia in the ambulatory setting: an analysis of 9,038 blocks. Anesthesiology. 2005;102(1):181-7.

52. Milan ZTD, McConnell P, Pickering J, et al. Three different approaches to transversus abdominis planeblock: a cadaveric study. Med Glas (Zenica). 2011;8(2):181-4.

53. Karima Karam Khan RIK. Analgesic effect of bilateral subcostal TAP block after laparoscopic cholecystectomy. J Ayub Med Coll Abbottabad. 2018;30(1):12-15

54. Kissin I. Preemptive analgesia. Anesthesiology. 2000;93:1138-43.

55. Jarman J, Marks N, Fahy CJ, et al. Anaesthetists' risk assessment of placebo nerve block studies using the SHAM (Serious Harm and Morbidity) scale. Anaesthesia. 2012;67(4):361-6.

Publisher's Note Springer Nature remains neutral with regard to jurisdictional claims in published maps and institutional affiliations. 